\title{
Capsaicin Inhibits Migration and Invasion via Inhibiting Epithelial-Mesenchymal Transition in Esophageal Squamous Cell Carcinoma By Up- Regulation of Cldn3 Expression
}

\section{Ji Feng}

The Army Medical University

\section{Yin Xu}

The Army Medical University

\section{Zhouling Wei}

The Army Medical University

Yiju Xia

The Army Medical University

Haoxiang Zhang

The Army Medical University

\section{Caifei Shen}

The Army Medical University

\section{Pu Wang}

The Army Medical University

\section{Wu Ran}

The Army Medical University

\section{Dianchun Fang}

The Army Medical University

\section{Yu Fang ( $\nabla$ fangyucs@sina.com )}

First Affiliated Hospital of Chongqing Medical University

\section{Research Article}

Keywords: esophageal squamous cell carcinoma, claudin 3, capsaicin, epithelial-mesenchymal transition, invasion and migration, lymph node metastasis

Posted Date: April 27th, 2021

DOI: https://doi.org/10.21203/rs.3.rs-437872/v1 
License: (c) (i) This work is licensed under a Creative Commons Attribution 4.0 International License. Read Full License 


\section{Abstract}

Purpose To investigate the effects of capsaicin on migration and invasion of esophageal squamous cell carcinoma and the roles of claudin-3 and epithelial-mesenchymal transition (EMT).

Methods Cldn3 expression was detected in paired cancerous and adjacent normal esophageal tissues by IHC. The association of Cldn3 with clinical data was analyzed. The migration and invasion of ESCC cells were investigated by exposure of capsaicin. The migration and invasion of ESCC cells with Cldn3 silencing or Cldn3 overexpression were also evaluated. The effects of capsaicin on lung metastasis of ESCC with or without Cldn3 silencing were evaluated.

Results: The positive expression of Cldn3 was associated with better prognosis of ESCC patients. Capsaicin attenuated migration and invasion of ESCC and inhibited EMT in a dose-dependent manner. Cldn3 silencing enhanced migration and invasion and EMT in ESCC cells. Cldn3 overexpression inhibited migration and invasion and EMT, similar to exposure of capsaicin. Cldn3 silencing also attenuated inhibition of migration and invasion and EMT by exposure of capsaicin. Cldn3 silencing attenuated inhibition of lung metastasis of ESCC by exposure of capsaicin in vivo.

Conclusion The positive expression of Cldn3 showed a better prognosis of ESCC, which was a potentially prognostic indicator. Capsaicin inhibited migration and invasion of ESCC through up-regulation of Cldn3 and inhibition of EMT.

\section{Introduction}

Esophageal cancer was the sixth leading cause of cancer-related death worldwide and the fourth leading cause of cancer-related death in China ${ }^{1,2}$. The mortality of esophageal cancer in China approximated $14.71 / 100,000$ in 2013 based on National Central Cancer Registry of China ${ }^{3}$. Despite advances in multimodality therapy, the prognosis of ESCC remained poor with an average five-year survival less than $30 \% 4$. The poor prognosis was partially led by locoregional invasion or distant metastasis at initial diagnosis. Locoregional recurrence and distant metastasis remained the primary cause of cancer-related death in ESCC patients after curative surgery ${ }^{9}$. Therefore, a better understanding of metastasis and identification of molecular targets for treatment were urgent for ESCC intervention.

Tight junction $\triangle T J \bigotimes$ was the most important intercellular junction of epithelial tissues, known as functioning in intercellular adhesion, cellular polarity and permeability of ion and macro molecular across epithelial barrier ${ }^{10,11}$. Tight junction proteins, physiologically existing among normal differentiated epithelial cells, were often lost in tumor tissues. Tumor cells were frequently featured by dysregulation of TJ function, less differentiation and aberrant cellular polarity ${ }^{12,13}$. Claudins, which belonged to the tetraspan transmembrane protein family, were the major constituents of TJ. Cldn3 was important for TJ formation, paracellular permeability and epithelial barrier function. Cldn3 was overexpressed in a variety of tumor tissues ${ }^{14}$. However, lower expression of Cldn3 achieved poor prognosis in certain types of 
tumors including ESCC 15-17. The methylation of CLDN3 tended to increase in squamous dysplasia and ESCC comparing with normal esophageal epithelium, contributing to lower expression of Cldn3 in preneoplastic and ESCC ${ }^{18,19}$. Loss of Cldn3 expression was associated with distant metastasis and the lower apoptotic index of ESCC ${ }^{17}$.

Epithelial-mesenchymal transition (EMT), which was the principal driver of metastasis and invasion of tumor cells, was observed in a variety of tumors including esophageal squamous cell carcinoma (ESCC). EMT prompted epithelial phenotype trans-differentiating into mesenchymal phenotype, which enable tumors cells to penetrate basement membrane and move to locoregional lymph nodes or distant organs 20. Loss of E-cadherin was a hallmark of EMT in epithelial tumors, which was more prone to metastasis 21,22. EMT markers played an important role in invasion and metastasis, which was closely related to prognosis ${ }^{23}$. Therefore, EMT markers were potential markers for diagnosis and therapy. It was reported Cldn3 could maintain the homeostasis of E-cadherin in ovarian cancer cells ${ }^{24}$.

Capsaicin was a naturally occurring alkaloid responsible for spices, food additives and drugs ${ }^{25}$, and had extensive pharmacological effects, e.g., pain killing, anti-inflammation and anti-oxidation. Recently, several studies indicated capsaicin also had anti-tumor effects through inhibiting proliferation and angiogenesis of tumor tissues ${ }^{26,27}$. Capsaicin was known to regulate cytoskeleton and tight junctions of epithelial and endothelial cells. Capsaicin was able to irreversibly open tight junction of microvascular endothelial cells, which potentially increased the permeability of macromolecular across blood-brain barrier ${ }^{28}$. Capsaicin also induced reversible opening of TJ through change of cytoskeleton proteins (e.g., Cofilin, F-actin) and tight junction proteins (e.g., Occludin) in epithelial cells ${ }^{29-32}$.

The effects of capsaicin on Cldn3 expression and invasion in ESCC were unclear. In this study, the association between Cldn3 expression and prognosis in ESCC was evaluated. The effects of capsaicin on migration and invasion of ESCC via Cldn3 and EMT dependent mechanism were investigated. ESCC cell lines including TE-1, TE-10, KYSE150 and ECa109 were used to address this issue.

\section{Methods}

\section{Patients and samples}

Paraffin-embedded sections of ESCC tissues and adjacent normal tissues were prepared from 93 ESCC patients (71 males and 22 females, average age 64.1 \pm 6.2-year-old) under radical esophagectomy from 2013 to 2014. None of the patients received neoadjuvant therapy before surgery. The tissue samples from primary tumor and regional lymph nodes were histologically examined with hematoxylin-eosin staining. The staging was confirmed according to International Union Against Cancer TNM classification 53. The differentiation was histologically subdivided into three levels: well-, moderate- and poor differentiation. The patients dying of surgery or causes other than esophageal cancer was excluded from this research. Demographic data including age, gender, stage, differentiation and histopathological factors were recorded and analyzed. All tissue samples were fixed in $10 \%$ formalin and embedded in 
paraffin wax. 4- $\mu \mathrm{m}$ serial sections were sliced and examined by immunohistochemistry (IHC). All animal experiments were approved by the Institutional Animal Care and Use Committee in Southwest Hospital of The Army Military Medical University and The First Affiliated Hospital of Chongqing Medical University. All the authors complied with the ARRIVE guidelines. All the animal experiments were performed in accordance with guidelines of animal care in Chongqing Medical University and The Army Medical University. All human experiments were approved by Ethical Committee in Southwest Hospital of The Army Military Medical University and The First Affiliated Hospital of Chongqing Medical University. The informed consent was obtained from all participants. All human experiments were performed in accordance with the guidelines of Helsinki Declaration.

\section{IHC staining}

The expression and distribution of Cldn3 was examined by IHC staining. Rabbit anti-Cldn3 polyclonal antibody (diluted 1:200; Abcam Inc区USA) was applied to detect Cldn3 expression. The semi-quantification of Cldn3 staining intensity was scored as follows: staining intensity was graded as 0 (negative), 1 (weak), 2 (moderate) and 3 (strong). The proportion of tumor cells with positive staining was graded as $0(<5 \%), 1$ (5-25\%), 2 (26-50\%), 3 (51-75\%) and 4 (>75\%). A final score was achieved by multiplying the two scores. Final score of 0-4 was defined as negative expression and score of 5-12 as positive expression 20 .

\section{Cell culture and protein extraction}

Human ESCC cell lines including TE-1, TE-10, KYSE150 and Eca109 were cultured in RPMI 1640 medium supplemented with $10 \%$ fetal calf serum (FCS). At the indicated time point, total protein was extracted using cell lysis buffer (Beyotime Biotechnology, China).

\section{RNA Interference}

Three target siRNA sequences were selected from different loci of human CLDN3. First, the most effective siRNA against human CLDN3 were screened. The optimal small hairpin RNA (shRNA) cassette targeting human CLDN3 (CCGGCGACCGCAAGGACTACGTCTACTC_GAGTAGACGTAGTCCTTGCGGTCGTTTTTG) and a scrambled sequence (RiboBio Co. Ltd, Guangzhou, China) was transfected into TE-1 and Eca109 cell lines in 6-well plates using jetPRIME transfection reagent (Polyplus-Transfection, IllkirchGraffenstaden, France) according to the manufacturer's instructions. Transfected cells were treated with $200 \mu \mathrm{M}$ capsaicin and control medium. To confirm the efficacy of siRNA CLDN3, Cldn3 expression in transfected cells were measured by RT-PCR and Western Blot.

\section{Lentiviral-mediated overexpression of Cldn3}

The cDNA coding full-length human CLDN3 was amplified by PCR and cloned into the lentiviral vector pGag/Pol (Gene Pharma Co., CHN) (pLV-CLDN3). To produce a negative control virus (LV-NC), a short noncoding sequence was cloned into the lentiviral pGag/Pol and processed in parallel with the target gene. TE- 1 and Eca109 cells $\left(3 \times 10^{5}\right)$ were transduced with pLV-CLDN3 and LV-NC using polybrene $(5 \mu \mathrm{g}$ 
$/ \mathrm{ml}$ ). Transduced cells were treated with $200 \mu \mathrm{M}$ capsaicin or control medium. To confirm the efficacy of pLV-CLDN3, Cldn3 expression in transduced cells were measured by RT-PCR and Western Blot.

\section{Western blotting}

Target proteins were separated by sodium dodecyl sulfate polyacrylamide gel electrophoresis and electrotransferred to a nitrocellulose membrane (Bio-Rad, USA). Western blot was carried out by sequential incubation in 5\% non-fat milk blocking buffer at room temperature for $60 \mathrm{~min}$, using the following antibodies: rabbit anti-Cldn3 polyclonal antibody (1:2000, Abcam, USA), rabbit anti-E-cadherin monoclonal antibody (1:1000, Millipore, USA), rabbit anti-N-cadherin monoclonal antibody (1:1000, Millipore, USA), rabbit anti-Vimentin monoclonal antibody (1:2000, Millipore, USA). Following overnight incubation at $4^{\circ} \mathrm{C}$, the secondary antibody was added and incubated for $60 \mathrm{~min}$ at $4^{\circ} \mathrm{C}$. HRP-GAPDH (Kangchen, China) was applied as loading control.

\section{RT-PCR}

Total RNA was isolated and cDNA was reversal transcribed according to the manufacturer's protocol. The mRNAs of target genes were measured by RT-PCR and analyzed by Rotor-Gene 3000 system (Corbett Research, Australia). Negative control was applied in each assay to ensure no contamination. All samples were detected in triplicate. The primer sequences were listed in Table 1.

\section{Cell migration by wound healing assay}

ESCC cell lines including KYSE150, TE-1, TE-10 and Eca109 were grown to confluence on 6-well plates and wounded by removing about $400 \mu \mathrm{m}$-wide strips of cells across the well with a standard $200 \mu \mathrm{l}$-pipette tip. After washing with HBSS, fresh culture medium was added and cells were incubated at $37^{\circ} \mathrm{C}$ in a humid environment with $5 \% \mathrm{CO}_{2}$. Wound closure was photographed every 15 mins by Nikon Eclipse TiS/L 100 microscope system. The experiments were performed twice and assayed in triplicate.

\section{Cell invasion by Transwell assay}

Cell invasion was evaluated using a Transwell chamber assay (Costar, USA). ESCC cell lines including TE1, TE-10, Eca109, KYSE150 were serum starved for $24 \mathrm{~h}$. Transwell inserts (BD Biosciences, USA) of 8mmpore size were coated with $150 \mathrm{ml}$ of 1:60 diluted Matrigel (BD Biosciences, USA). Cells $\left(1 \times 10^{5}\right)$ were plated onto the top of each coated filters in $150 \mathrm{ml}$ serum-free medium. $300 \mathrm{ml}$ of the same medium containing $20 \%$ FCS was placed in the lower chamber to act as a chemoattractant. After $24 \mathrm{~h}$ incubation at $37^{\circ} \mathrm{C}$, cells which did not move across the pores of Transwell inserts were manually removed with cotton swabs. The inserts were fixed in cold methanol for 10 mins followed by staining with $0.01 \%$ crystal violet in $20 \%$ ethanol. Cell counting was performed by Coomassie blue staining followed by visualizing under microscope (Leica, Germany). The experiments were performed twice and assayed in triplicate.

\section{Tail vein metastatic assay}


The tail vein metastatic assay was performed as previously described ${ }^{54}$. TE- 1 cells $\left(1 \times 10^{6}\right)$ treated with capsaicin $200 \mu \mathrm{M}$ or siRNA Cldn3 plus capsaicin $200 \mu \mathrm{M}$ were injected intravenously into nude mice through tail vein. The mice were sacrificed at 8 weeks after treated TE-1 cells injection. The metastatic lesions of lungs were harvested and examined with histology. The number of metastatic foci from lungs were quantified. Eight mice were included in each group.

\section{Statistical Analysis}

For the data from histologic analysis, comparisons were assessed using the chi-squared test. Survival curve was performed by Kaplan-Meier method. The discrepancies in survival distributions were evaluated by log-rank test. Cox's proportional hazards modeling was performed to confirm factors influencing on survival. For the data of RT-PCR and Western blotting, comparisons between groups were assessed by one-way ANOVA followed by Bonferroni's test. The data were presented as the means \pm SD. Statistical significance was estimated using SPSS version 19.0 software (SPSS, Inc., Chicago, IL, USA). $\mathrm{P}<0.05$ was considered to a significant difference.

\section{Results}

\section{The association of Cldn3 expression with clinical data in ESCC patients}

Cldn3 was primarily located at cellular membrane and cytoplasm of ESCC cells (Figure 1A-C), while negative or mild staining of Cldn3 was observed in adjacent normal tissues of ESCC. The positive expression of Cldn3 accounted for $43 \%$ of ESCC tissues (40/93). It was indicated that negative expression of Cldn3 was associated with locoregional lymph node metastasis and poor survival $(P<0.05$; Table2). There was no significant association among Cldn3 expression and gender, age, location, differentiation, tumor size and T status $(P>0.05$; Table 2$)$. The median follow-up was 25.6 months ranging from 1 to 52 months. Kaplan-Meier analysis revealed the median survival time for ESCC patients with positive expression of Cldn3 was 38.0 months, whereas 24.1 months for patients with negative expression of Cldn3 ( $\mathrm{P}<0.05$; Figure 1E). It was indicated ESCC patients with negative expression of Cldn3 achieved poor prognosis $(P<0.05$; Table 2$)$.

\section{Effects of capsaicin on migration and invasion of ESCC cells}

Four ESCC cell lines including TE-1, TE-10, Eca109 and KYSE150 were treated with different doses $(0 \mu \mathrm{M}$, $100 \mu \mathrm{M}$ and $200 \mu \mathrm{M}$ ) of Capsaicin for $48 \mathrm{~h}$ respectively. The spreading speed of TE-1, TE-10, Eca109 and KYSE150 cells with Capsaicin administering was significantly slower than that in control cells by wound healing assay $(p<0.05$; Figure $2 A-B)$. The average number of invading cells treated with capsaicin was significantly reduced as compared with that of control cells by Transwell assay $(p<0.05$; Figure $2 C-D)$.

\section{Effects of capsaicin on Cldn3 and EMT markers expression in ESCC cells}


ESCC cells including TE-1, TE-10, Eca109 and KYSE150 were exposed to $0 \mu \mathrm{M}, 100 \mu \mathrm{M}, 200 \mu \mathrm{M}$ capsaicin for $12 \mathrm{~h}$ respectively. The expression of Cldn3 mRNA and protein was detected. The expression of Cldn3 mRNA and protein was up-regulated by exposure of capsaicin in a dose-dependent manner $(P<0.05$; Figure $3 \mathrm{~A}-\mathrm{C}$ ). ESCC cells exposed by capsaicin showed up-regulation of E-cadherin and down-regulation of $\mathrm{N}$-cadherin and vimentin in a dose-dependent manner $(\mathrm{P}<0.05$; Figure3A-C).

\section{Effects of capsaicin on migration and invasion of ESCC cells via Cldn3- and EMT-dependent mechanisms}

TE-1 and Eca109 cells were selected for Cldn3 RNA interference based on higher endogenous expression of Cldn3 protein (Figure 3C). CLDN3 gene silencing contributed to aggravated invasion and migration of both ESCC cells $(\mathrm{P}<0.05$; Figure 4A-B). ESCC cells with CLDN3 gene silencing showed increased expression of $\mathrm{N}$-cadherin and Vimentin and decreased expression of E-cadherin comparing with control ESCC cells, suggesting augmented EMT $(P<0.05$; Figure 4C-D). However, capsaicin failed to attenuate the aggravated migration and invasion and EMT induced by Cldn3 inhibition ( $P>0.05$; Figure 4A-D).

KYSE150 and TE-10 cell lines were selected for Cldn3 overexpression assay based on lower endogenous expression of Cldn3 protein (Figure 3C). ESCC cells with Cldn3 overexpression plus capsaicin exposure showed inhibitory migration and invasion similar to those with capsaicin exposure alone $(P>0.05$; Figure 5A-B). ESCC cells with Cldn3 overexpression showed upregulation of E-cadherin and downregulation of $\mathrm{N}$-cadherin and Vimentin, which was similar to the cells with Cldn3 overexpression plus capsaicin exposure ( $P>0.05$; Figure $5 C-D)$ and suggested attenuated EMT ( $P<0.05$; Figure $5 C-D)$. It was suggested capsaicin attenuated migration and invasion of ESCC cells via Cldn3 overexpression and EMT inhibition.

\section{Inhibitory effect of capsaicin on lung metastasis of ESCC}

To further explore the effect of capsaicin on metastasis of ESCC in vivo, tail vein metastatic assay was performed. The number of lung metastatic nodules in TE-1 cells treated with $200 \mu \mathrm{M}$ capsaicin exposure was significantly lower than that in TE-1 cells $(P<0.05$; Figure 6A-B). Lung metastatic nodules in TE- 1 cells treated with Cldn3 SiRNA plus $200 \mu \mathrm{M}$ capsaicin exposure outnumbered those in TE-1 cells with $200 \mu \mathrm{M}$ capsaicin exposure $(P<0.05$; Figure $6 A-B)$. It was indicated capsaicin inhibited lung metastasis of ESCC by overexpression of Cldn3.

\section{Discussion}

It was reported capsaicin possessed the properties of anti-tumor and anti-proliferation; however, the exact mechanism was unknown yet. In this study, it was suggested capsaicin inhibited invasion and migration of human ESCC cells via Cldn3- and EMT-dependent mechanism. It was provided a theoretical basis for capsaicin as a potential anti-tumor medicine to ESCC.

Intercellular junction proteins played a key role in the process of progress and metastasis of tumors. Normal epithelial cells were linked by tight junctions (TJ), adheren junctions, gap junctions, desmosomes, and etc. Cldn3 was an important structural protein to participate in TJ formation, which maintained 
homeostasis of epithelial and endothelial barrier function ${ }^{33-35}$. The expression of Cldn3 was also associated with invasion and prognosis of epithelial tumors. Low expression of Cldn3 was reported to be significantly associated with poor prognosis of hepatocellular carcinoma and lung squamous cell carcinoma, which was caused by promoting cell motility, invasion and metastasis ${ }^{36,37}$. High expression of Cldn3 in lung adenocarcinoma indicated poor survival and cancer progression ${ }^{38}$. The discrepancy of Cldn3 expression might be explained by histological heterogeneity of tumors. Cldn3 was also considered as a potential blood-based biomarker in prostate cancer ${ }^{39}$. In this study, low expression of Cldn3 was associated with a poor prognosis of ESCC (Figure 1D).

Capsaicin was a kind of pungent ingredient extracted from red hot pepper. Capsaicin possessed properties of anti-tumor and pro-apoptosis in some tumors. Capsaicin efficiently induced apoptosis in hepatocellular carcinoma via acceleration of intracellular $\mathrm{Ca}^{2+}$ production, activation of reactive oxygen species (ROS) and regulation of Bcl-2 and caspase-3 ${ }^{40,41}$. Capsaicin also induced programmed cell death of colon cancer cells via activation of ROS and regulation of PPARY, Bcl-2 and caspases ${ }^{\mathbf{4 2 , 4 3}}$. However, the effect of capsaicin on migration and invasion of human ESCC cells was still unclear. In this study, capsaicin significantly inhibited migration and invasion of ESCC cells via regulation of Cldn3 and EMT, which was a potential preventive and therapeutic agent for ESCC treatment.

EMT was the process by which polarized epithelial cells that were connected via adhesion lose their characteristics and acquired migratory and invasive properties characteristic of a mesenchymal cells 44,45. Cells under EMT displayed loss of epithelial cell markers (e.g., E-cadherin) and gain of mesenchymal cell markers (e.g., N-cadherin and Vimentin). EMT promoted migration and invasion of epithelial cells, accelerated metastasis of cancer cells and achieved acquisition of stem cell-like properties ${ }^{46-50}$. In this study, capsaicin inhibited migration and invasion of ESCC cells via attenuating EMT.

Capsaicin was a known anti-tumor agent through interfering with signal pathways, e.g., NFkB and Wnt pathway ${ }^{51,52}$. However, there was little known about the relationship between capsaicin exposure and Cldn3 expression. In this study, capsaicin triggered activation of Cldn3 expression. CLDN3 gene silencing led to down-regulation of E-cadherin and up-regulation of N-cadherin and Vimentin. In contrast, Overexpression of Cldn3 contributed to up-regulation of E-cadherin and down-regulation of $\mathrm{N}$-cadherin and Vimentin. It was suggested capsaicin inhibited migration and invasion of ESCC via Cldn3- and EMTdependent manner.

In conclusion, low expression of Cldn3 was associated with poor prognosis of ESCC patients. Cldn3 was a potential prognostic indicator of ESCC. Capsaicin inhibited invasion and metastasis of ESCC via Cldn3 overexpression and inhibition of EMT. Capsaicin was a potential anti-tumor agent for ESCC treatment.

\section{Abbreviations}

ESCC esophageal squamous cell carcinoma 
Cldn3 claudin-3

EMT epithelial-mesenchymal transition

TJ tight junction

IHC immunohistochemistry

ROS reactive oxygen species

\section{Declarations}

\section{Acknowledgement}

This study was supported by grants from the National Science Foundation of China (No. 81570476 and 81400590)

\section{Author contributions}

Fang DC and Fang $\mathrm{Y}$ had the idea for the article; Feng $\mathrm{J}$ and $\mathrm{Xu} Y \mathrm{Y}$ performed the literature search and data analysis; Shen CF, Wang P, Wu R and Xia YJ performed cell culture, transfection, QPCR, WB, etc.; Fang Y, Zhang HX and Wei ZL drafted and/or critically revised the work.

\section{Competing Interests Statements}

There is no conflict of interest to declare for all authors

\section{Ethic declaration}

Placed in the Methods Section

\section{Consent to participate}

Informed consent was obtained from all individuals included in this study

\section{Consent to publish}

The participant has consented to the submission of this study to this journal

\section{Data availability}

The datasets generated during the current study are available from the corresponding author on reasonable request

\section{References}


1. Bray, F., Ren, J. S., Masuyer, E. \& Ferlay, J. Global estimates of cancer prevalence for 27 sites in the adult population in 2008. Int $J$ Cancer. 132, 1133-1145 (2013). )

2. Di Pardo, B. J. et al. The Global Burden of Esophageal Cancer: A Disability-Adjusted Life-Year Approach. World J of surg 40, 395-401, https://doi.10.1007/s00268-015-3356-2(2016).

3. Zheng, R., Zeng, H., Zhang, S. \& Chen, W. Estimates of cancer incidence and mortality in China, 2013. Chin J Cancer 36, 66, https://doi.10.1186/s40880-017-0234-3(2017).

4. Worni, M. et al. Does surgery improve outcomes for esophageal squamous cell carcinoma? An analysis using the surveillance epidemiology and end results registry from 1998 to 2008. J Am Coll Surg 215, 643-651, https://doi.10.1016/j.jamcollsurg. 2012.07 .006 (2012).

5. Gallo, A. \& Cha, C. Updates on esophageal and gastric cancers. World J of gastroentero/ 12, 32373242, https://doi.10.3748/wjg.v12.i20.3237 (2006).

6. Ando, N., Ozawa, S., Kitagawa, Y., Shinozawa, Y. \& Kitajima, M. Improvement in the results of surgical treatment of advanced squamous esophageal carcinoma during 15 consecutive years. Ann Surg 232, 225-232, https://doi.10.1097/00000658.200008000.00013(2000).

7. Adham, M. et al. Combined chemotherapy and radiotherapy followed by surgery in the treatment of patients with squamous cell carcinoma of the esophagus. Cancer. 89, 946-954 (2000).

8. Refaely, Y. \& Krasna, M. J. Multimodality therapy for esophageal cancer. Surg clin North Am 82, 729746, https://doi.10.1016/s0039-6109(02)00029-4(2002).

9. Vallbohmer, D., Brabender, J., Metzger, R. \& Holscher, A. H. Genetics in the pathogenesis of esophageal cancer: possible predictive and prognostic factors. J Gastrointest Surg 14, S75-80, https://doi.10.1007/s11605-009-1021-5(2010).

10. Furuse, M., Sasaki, H. \& Tsukita, S. Manner of interaction of heterogeneous claudin species within and between tight junction strands. J Cell Biol 14, 891-903, https://doi.10.1083/jcb.147.4.891(1999).

11. Matsuda, M., Kubo, A., Furuse, M. \& Tsukita, S. A peculiar internalization of claudins, tight junctionspecific adhesion molecules, during the intercellular movement of epithelial cells. J Cell Sci 117, 1247-1257. https://doi.10.1242/jcs.00972(2004).

12. Weinstein, R. S., Merk, F. B. \& Alroy, J. The structure and function of intercellular junctions in cancer. Adv Cancer Research 23, 23-89, https://doi.10.1016/s0065-230x(08)60543-6(1976).

13. Soler, A. P. et al. Increased tight junctional permeability is associated with the development of colon cancer. Carcinogenesis 20, 1425-1431, https://doi.10.1093/carcin/20.8.1425(1999).

14. Rangel, L. B. et al. Tight junction proteins claudin-3 and claudin-4 are frequently overexpressed in ovarian cancer but not in ovarian cystadenomas. Clin Cancer Res. 9, 2567-2575 (2003).

15. Szasz, A. M. et al. Identification of a claudin-4 and E-cadherin score to predict prognosis in breast cancer. Cancer Sci 102, 2248-2254, https://doi.10.1111/j.1349-7006. 2011.02085.x (2011).

16. Shang, X., Lin, X., Alvarez, E., Manorek, G. \& Howell, S. B. Tight junction proteins claudin-3 and claudin-4 control tumor growth and metastases. Neoplasia 14, 974-985, 
https://doi.10.1593/neo.12942(2012).

17. Takala, H., Saarnio, J., Wiik, H. \& Soini, Y. Claudins 1, 3, 4, 5 and 7 in esophageal cancer: loss of claudin 3 and 4 expression is associated with metastatic behavior. APMIS 115, 838-847, https://doi.10.1111/j.1600-0463 2007.apm_656.x (2007).

18. Roth, M. J. et al. p16, MGMT, RARbeta2, CLDN3, CRBP and MT1G gene methylation in esophageal squamous cell carcinoma and its precursor lesions. Oncol Rep 15,1591-1597(2006).

19. Adams, L. et al. Promoter methylation in cytology specimens as an early detection marker for esophageal squamous dysplasia and early esophageal squamous cell carcinoma. Cancer Prev Res (Phila) 1, 357-361, https://doi.1158/ 1940-6207.CAPR-08-0061 (2008).

20. Huo, X. et al. Prognostic significance of the epithelial-mesenchymal transition factor zinc finger Ebox-binding homeobox 2 in esophageal squamous cell carcinoma. Oncol Lett 14, 2683-2690, https://doi.10.3892/ol. 2017.6559 (2017).

21. Polyak, K. \& Weinberg, R. A. Transitions between epithelial and mesenchymal states: acquisition of malignant and stem cell traits. Nat Rev Cancer 9, 265-273, https://doi.10.1038/nrc2620(2009).

22. Yilmaz, M. \& Christofori, G. EMT, the cytoskeleton, and cancer cell invasion. Cancer Metastasis Rev 28, 15-33, https://doi.10.1007/s10555-008-9169-0(2009).

23. Peinado, H., Olmeda, D., Cano, A. \& Snail Zeb and bHLH factors in tumour progression: an alliance against the epithelial phenotype? Nat Rev Cancer 7, 415-428, https://doi.10.1038/nrc2131(2007).

24. Lin, X., Shang, X., Manorek, G. \& Howell, S. B. Regulation of the Epithelial-Mesenchymal Transition by Claudin-3 and Claudin-4. PloS one 8, e67496, https://doi.10.1371/journal.pone.0067496(2013).

25. Reyes-Escogido Mde, L., Gonzalez-Mondragon, E. G. \& Vazquez-Tzompantzi, E. Chemical and pharmacological aspects of capsaicin. Molecules 16, $1253-127$, https://doi.10.3390/molecules 16021253(2011).

26. Wu, C. C. et al. Capsaicin induced cell cycle arrest and apoptosis in human esophagus epidermoid carcinoma CE 81T/VGH cells through the elevation of intracellular reactive oxygen species and $\mathrm{Ca} 2$ + productions and caspase-3 activation. Muta Res 601, 71-82, https://doi.10.1016/j.mrfmmm. 2006.06.015 (2006).

27. Kim, J. D. et al. Capsaicin can alter the expression of tumor forming-related genes which might be followed by induction of apoptosis of a Korean stomach cancer cell line, SNU-1. Cancer Lett 120, 235-241, https://doi.10.1016/s0304-3835(97)00321-2(1997).

28. Kaiser, M. et al. The Influence of Capsaicin on the Integrity of Microvascular Endothelial Cell Monolayers. Int J Mol Sci 20, 122, https://doi.10.3390/ijms20010122(2018).

29. Kaiser, M., Chalapala, S., Gorzelanny, C., Perali, R. S. \& Goycoolea, F. M. The Effect of Capsaicin Derivatives on Tight-Junction Integrity and Permeability of Madin-Darby Canine Kidney Cells. $J$ Pharm Sci 10, 630-638, https://doi.10.1016/j.xphs. 2015.10.017(2016).

30. Nagumo, Y., Han, J., Arimoto, M., Isoda, H. \& Tanaka, T. Capsaicin induces cofilin dephosphorylation in human intestinal cells: the triggering role of cofilin in tight-junction signaling. Biochem Biophys Res Commun 355, 520-525, https://doi.10.1016/j.bbrc. 2007.02 .002 (2007). 
31. Nagumo, Y., Han, J., Bellila, A., Isoda, H. \& Tanaka, T. Cofilin mediates tight-junction opening by redistributing actin and tight-junction proteins. Biochem Biophys Res Commun 377, 921-925, https://doi.10.1016/j.bbrc. 2008.10.071(2008).

32. Shiobara, T., Usui, T., Han, J., Isoda, H. \& Nagumo, Y. The reversible increase in tight junction permeability induced by capsaicin is mediated via cofilin-actin cytoskeletal dynamics and decreased level of occludin. PLoS One 8, e79954, https://doi.10.1371/journal.pone.0079954(2013).

33. Berndt, P. et al. Tight junction proteins at the blood-brain barrier: far more than claudin-5. Cell Mol Life Sci 76, 1987-2002, https://doi.10.1007/s00018-019-03030-7(2019).

34. Leiz, J. \& Schmidt-Ott, K. M. Claudins in the Renal Collecting Duct. Int J Mol Sci 21, 221, https://doi.10.3390/ijms21010221(2019).

35. Deng, Z. et al. Claudin reduction may relate to an impaired skin barrier in rosacea. J Dermatol 46, 314-321, https://doi.10.1111/1346-8138.14792(2019).

36. Jiang, L. et al. CLDN3 inhibits cancer aggressiveness via Wnt-EMT signaling and is a potential prognostic biomarker for hepatocellular carcinoma. Oncotarget 5, 7663-7676, https://doi.10.18632/oncotarget.2288(2014).

37. Che, J. et al. Claudin-3 Inhibits Lung Squamous Cell Carcinoma Cell Epithelial-mesenchymal Transition and Invasion via Suppression of the Wnt/beta-catenin Signaling Pathway. Int J Med Sci 15, 339-351, https://doi.10.7150/ijms.22927(2018).

38. Zhang, L. et al. Claudin-3 expression increases the malignant potential of lung adenocarcinoma cells: role of epidermal growth factor receptor activation. Oncotarget 8, 23033-23047, https://doi.10. 18632/oncotarget.14974(2017).

39. Worst, T. S. et al. Database-augmented Mass Spectrometry Analysis of Exosomes Identifies Claudin 3 as a Putative Prostate Cancer Biomarker. Mol cell proteomics 16, 998-1008, https://doi.10 1074/mcp.M117.068577(2017).

40. Huang, S. P. et al. Capsaicin-induced apoptosis in human hepatoma HepG2 cells. Anticancer Res. 29, 165-174 (2009).

41. Jung, M. Y., Kang, H. J. \& Moon, A. Capsaicin-induced apoptosis in SK-Hep-1 hepatocarcinoma cells involves Bcl-2 downregulation and caspase-3 activation. Cancer Lett 165,139-145, https://doi.10.1016/s0304-3835(01)00426-8(2001).

42. Kim, C. S. et al. Capsaicin, a spicy component of hot pepper, induces apoptosis by activation of the peroxisome proliferator-activated receptor gamma in HT-29 human colon cancer cells. J Med food 7, 267-273, https://doi.10.1089/jmf. 2004.7 .267 (2004).

43. Lu, H. F. et al. Antitumor activity of capsaicin on human colon cancer cells in vitro and colo 205 tumor xenografts in vivo. J Agric Food Chem 58, 12999-13005, https://doi.10. 021/jf103335w(2010).

44. Kajiyama, H. et al. Chemoresistance to paclitaxel induces epithelial-mesenchymal transition and enhances metastatic potential for epithelial ovarian carcinoma cells. Int J of Oncol. 31, 277-283 (2007). 
45. Sun, L. et al. MiR-200b and miR-15b regulate chemotherapy-induced epithelial-mesenchymal transition in human tongue cancer cells by targeting BMI1. Oncogene 31, 432-445, https://doi.10.1038/onc. 2011.263 (2012).

46. Ewald, A. J., Brenot, A., Duong, M., Chan, B. S. \& Werb, Z. Collective epithelial migration and cell rearrangements drive mammary branching morphogenesis. Dev Cell14, 570-581, https://doi.10.1016/j.devcel. 2008.03.003 (2008).

47. Thiery, J. P., Acloque, H., Huang, R. Y. \& Nieto, M. A. Epithelial-mesenchymal transitions in development and disease. Cel/ 139, 871-890, https://doi.10.1016/j.cell. 2009.11.007 (2009).

48. Lopez-Novoa, J. M. \& Nieto, M. A. Inflammation and EMT: an alliance towards organ fibrosis and cancer progression. EMBO Mol Med 1, 303-314, https://doi.10. 1002/emmm.200900043(2009).

49. Keshamouni, V. G. \& Schiemann, W. P. Epithelial-mesenchymal transition in tumor metastasis: a method to the madness. Future Oncol 5, 1109-1111, https://doi.10. 2217/fon.09.87(2009).

50. Gomes, L. R., Terra, L. F., Sogayar, M. C. \& Labriola, L. Epithelial-mesenchymal transition: implications in cancer progression and metastasis. Curr Pharm Biotechnol 12, 1881-1890, https://doi.10. 2174/138920111798377102(2011).

51. Surh, Y. J., Han, S. S., Keum, Y. S., Seo, H. J. \& Lee, S. S. Inhibitory effects of curcumin and capsaicin on phorbol ester-induced activation of eukaryotic transcription factors, NF-kappaB and AP-1. Biofactors 12, 107-112, https://doi.10.1002/biof.5520120117(2000).

52. Lee, S. H., Richardson, R. L., Dashwood, R. H. \& Baek, S. J. Capsaicin represses transcriptional activity of beta-catenin in human colorectal cancer cells. J Nutr Biochem 23, 646-655, https://doi.10. 1016/j.jnutbio. 2011.03 .009 (2012).

53. Pramanik, K. C., Boreddy, S. R. \& Srivastava, S. K. Role of mitochondrial electron transport chain complexes in capsaicin mediated oxidative stress leading to apoptosis in pancreatic cancer cells. PloS one 6, e20151, https://doi.10.1371/journal.pone.0020151(2011).

54. Huang, Y., Yang, M., Yang, H. \& Zeng, Z. Upregulation of the GRIM-19 gene suppresses invasion and metastasis of human gastric cancer SGC-7901 cell line. Exp Cell Res 316, 2061-2070, https://doi.10.1016/j.yexcr. 2010.05.010 (2010).

\section{Tables}

Table 1 The primer sequences 


\begin{tabular}{|c|c|c|}
\hline Symbol & sense & antisense \\
\hline Cldn3 & 5'- САССАССАCCAACACCAC-3' & 5'-CAGGTATTGGCGGTCACC-3' \\
\hline E-cadherin & 5囚-ACAACGCCCCCATACCAGA-3' & 5囚-CACTCGCCCCGTGTGTTAGT-3’ \\
\hline N-cadherin & 5囚-CTGAGCCTCACCTGTGCGC-3’ & 5囚-CACTCGCCCCGTGTGTTAGT-3’ \\
\hline Vimentin & 5囚-AGCATCTCCTCCTGCAATTT-3’ & 5囚-AGGTGGACCAGCTAACCAAC-3' \\
\hline GAPDH & 5囚-ACCCAGAAGACTGTGGATGG-3' & 5囚-ACGCCTGCTTCACCACСТTC-3' \\
\hline
\end{tabular}

Table 2 The association between Cldn3 and clinical data in ESCC patients(by one-way ANOVA) 


\begin{tabular}{|c|c|c|c|c|c|}
\hline \multirow[t]{2}{*}{ Clinical data } & & \multirow[t]{2}{*}{$\mathrm{n}$} & \multicolumn{2}{|l|}{ Cldn3 } & \multirow[t]{2}{*}{ P-value } \\
\hline & & & positive & Negative & \\
\hline \multicolumn{6}{|l|}{ Sex } \\
\hline & Male & 71 & 29 & 42 & \multirow[t]{2}{*}{0.449} \\
\hline & Female & 22 & 11 & 11 & \\
\hline \multicolumn{6}{|l|}{ Age } \\
\hline & $<60$-year-old & 35 & 19 & 16 & \multirow[t]{2}{*}{0.088} \\
\hline & $>60$-year-old & 58 & 21 & 37 & \\
\hline \multicolumn{6}{|l|}{ Diameter } \\
\hline & $<5 \mathrm{~cm}$ & 62 & 27 & 35 & \multirow[t]{2}{*}{0.882} \\
\hline & $>5 \mathrm{~cm}$ & 31 & 13 & 18 & \\
\hline \multicolumn{6}{|l|}{ Differentiation } \\
\hline & Well & 15 & 8 & 7 & \multirow[t]{3}{*}{0.310} \\
\hline & Moderate & 63 & 28 & 35 & \\
\hline & Poorly & 15 & 4 & 11 & \\
\hline \multicolumn{6}{|l|}{ pT } \\
\hline & pT1-2 & 29 & 14 & 15 & \multirow[t]{2}{*}{0.490} \\
\hline & pT3-4 & 64 & 26 & 38 & \\
\hline \multicolumn{6}{|l|}{$\mathrm{pN}$} \\
\hline & pN1-2 & 44 & 14 & 30 & \multirow[t]{2}{*}{0.039} \\
\hline & pNO & 49 & 26 & 23 & \\
\hline \multicolumn{6}{|l|}{ Stage } \\
\hline & 1 & 4 & 4 & 0 & \multirow[t]{3}{*}{0.011} \\
\hline & $\|$ & 24 & 14 & 10 & \\
\hline & III & 65 & 10 & 55 & \\
\hline \multicolumn{6}{|l|}{ Prognosis } \\
\hline & Survival & 40 & 26 & 14 & \multirow[t]{2}{*}{0.024} \\
\hline & Mortality & 53 & 14 & 39 & \\
\hline
\end{tabular}


Figures
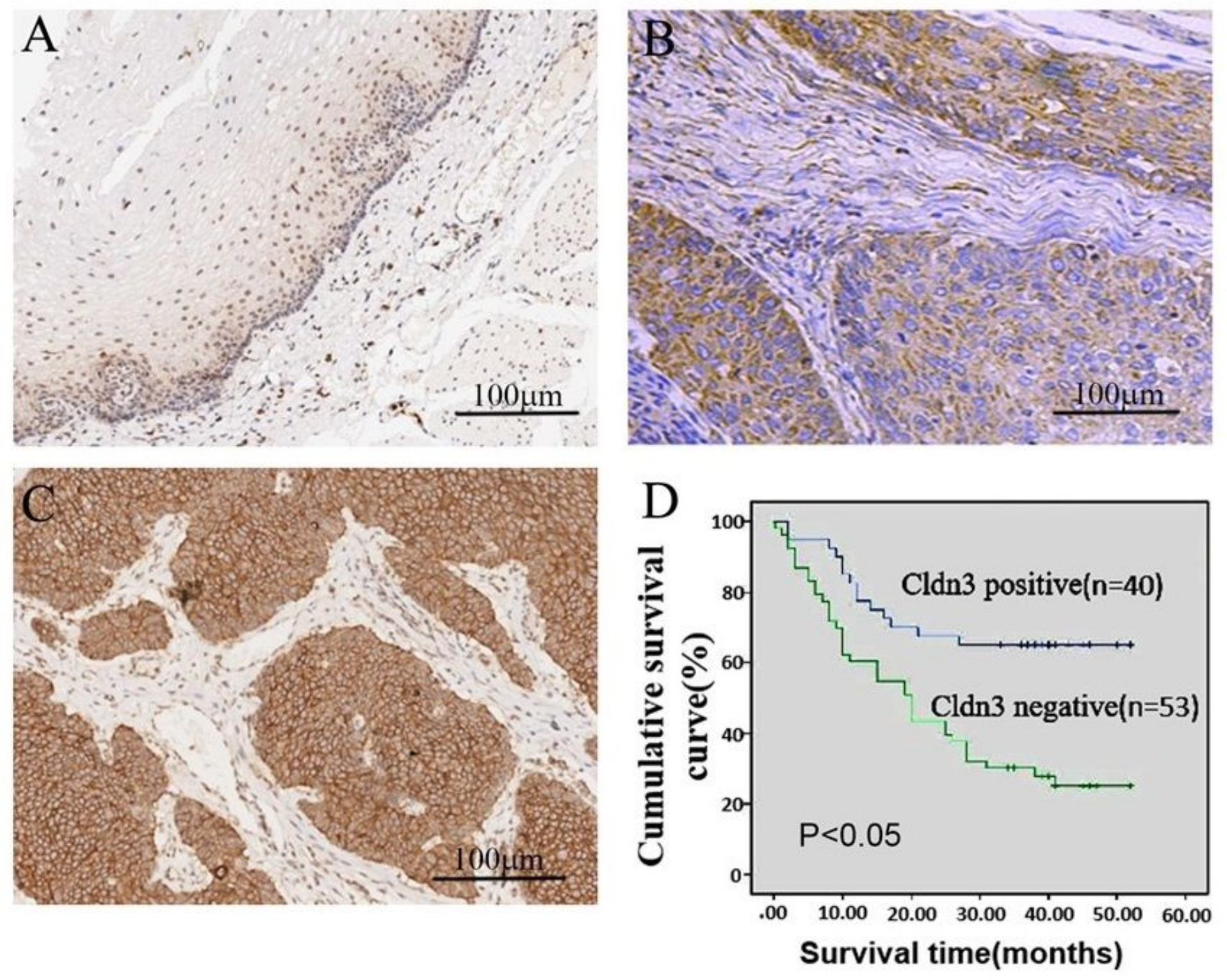

Figure 1

The association of Cldn3 expression with over survival (OS) in ESCC patients A) Negative or weak expression of Cldn3 in normal esophageal epithelial cells; B) Weak expression of Cldn3 in ESCC tissues; C) Strong expression of Cldn3 in ESCC tissues; D) Kaplan-Meier analysis of OS in ESCC patients with Cldn3 positive and negative. Scale bar equals to $100 \mu \mathrm{m}$. 
$\mathbf{A}$
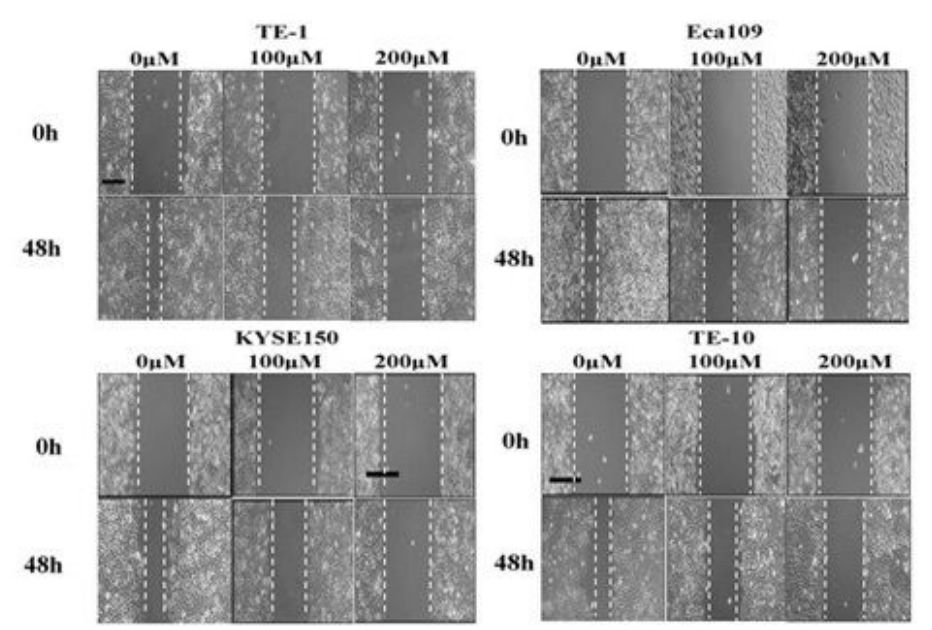

C

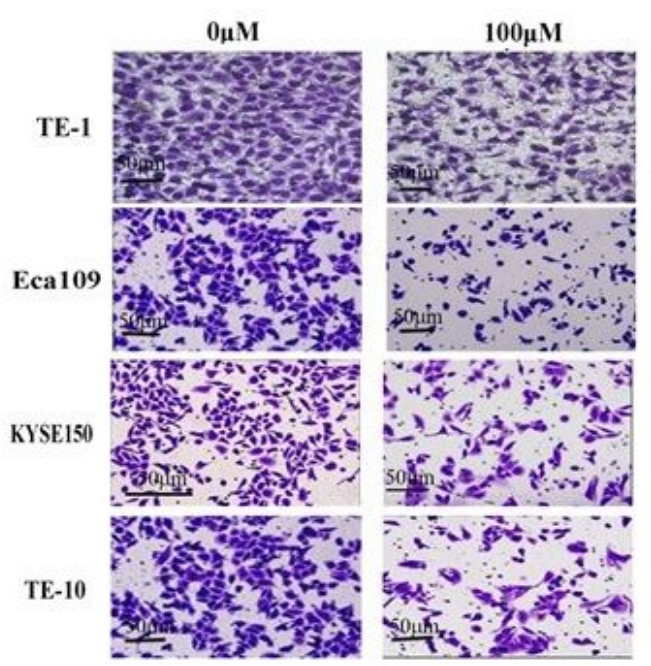

B

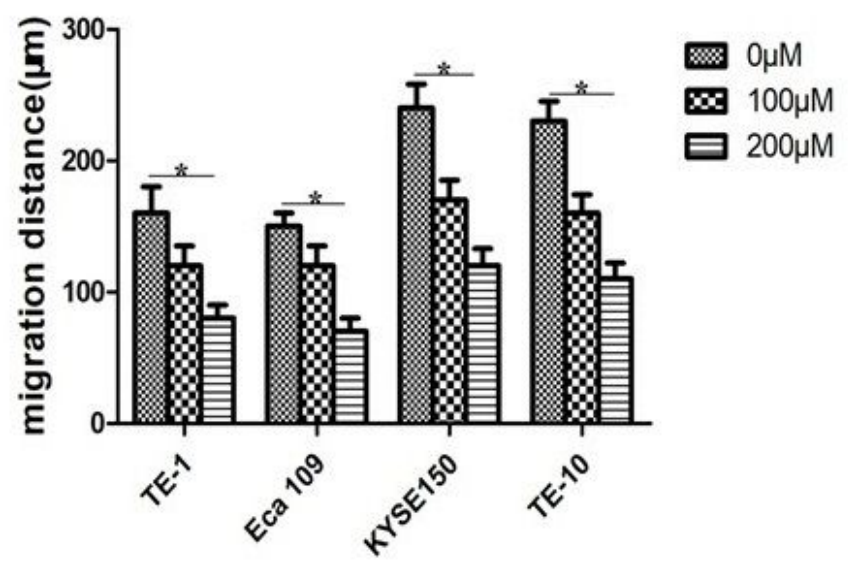

D

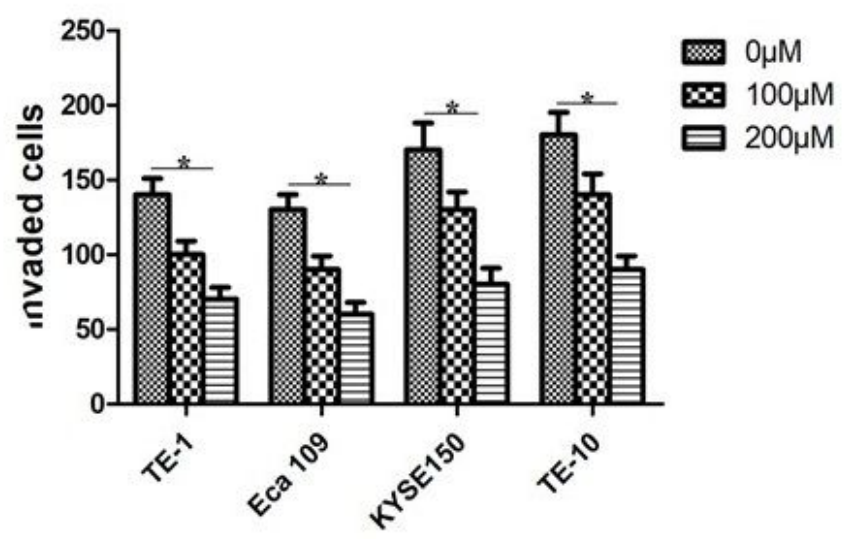

Figure 2

The effects of Capsaicin on migration and invasion of ESCC cells A) Comparison of migration distance of ESCC cell lines treated with capsaicin of different doses by wound healing assay; 2) Quantification of migration distance of ESCC cell lines treated with capsaicin of different doses by wound healing assay; 3) Comparison of invaded ESCC cells treated with capsaicin of different doses by Transwell assay; 4) Quantification of invaded ESCC cells treated with capsaicin of different doses by Transwell assay. All data were presented as mean \pm SD of three independent experiments. Statistical analysis was performed using an unpaired Student's t test. *, P<0.05. Scale bar equals to $50 \mu \mathrm{m}$. 

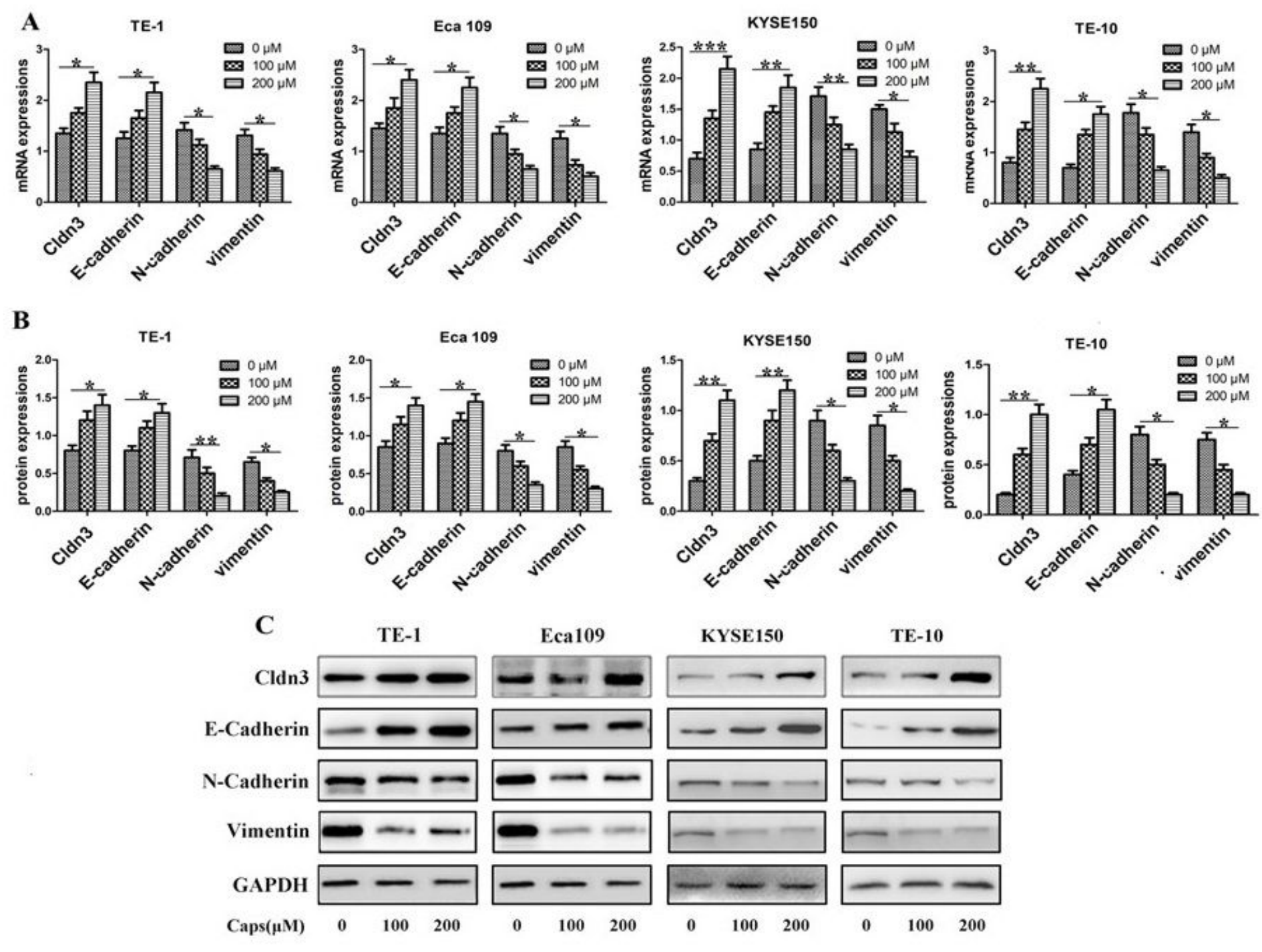

Figure 3

Effects of capsaicin on the expression of Cldn3 and Epithelial-Mesenchymal Transition (EMT) markers in ESCC cell lines A) Quantification of mRNA expression of Cldn3 and EMT markers in ESCC cell lines treated with capsaicin of different doses; B) Quantification of protein expression of Cldn3 and EMT markers in ESCC cell lines treated with capsaicin of different doses; C) Comparison of protein expression of Cldn3 and EMT markers in ESCC cell lines treated with capsaicin of different doses by Western Blotting. All data were presented as mean \pm SD of three independent experiments. Statistical analysis was performed using an unpaired Student's $t$ test. *, $P<0.05 ; * \star, P<0.01$. 

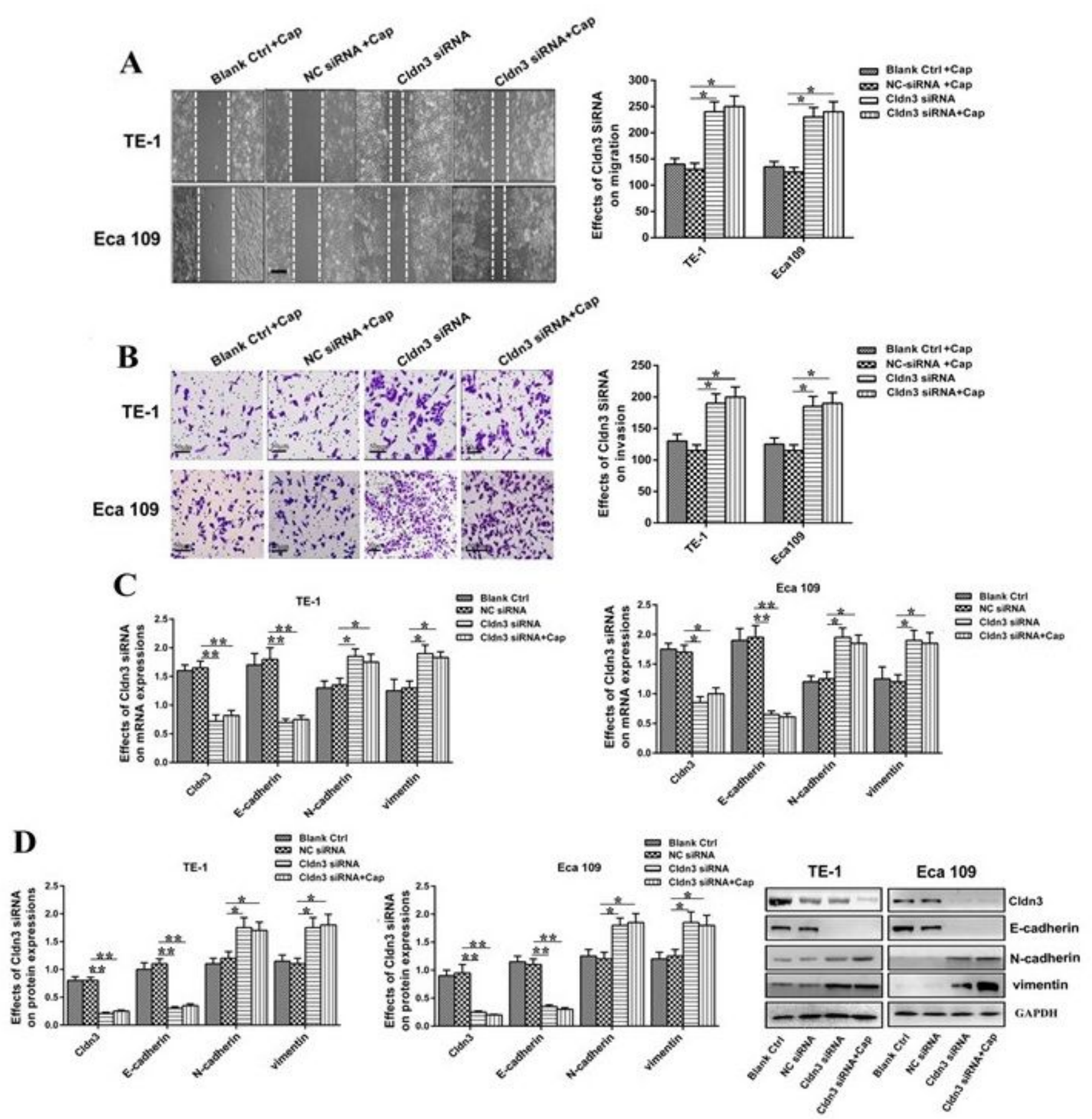

Figure 4

Effects of CLDN3 gene silencing on migration and invasion of ESCC cells and the expression of EMT markers A) Comparison of migration distance of ESCC cells treated with CLDN3 gene silencing or CLDN3 gene silencing plus capsaicin by wound healing assay; B) Comparison of invaded ESCC cells treated with CLDN3 gene silencing or CLDN3 gene silencing plus capsaicin by Transwell assay; C) Quantification of mRNA expression of Cldn3 and EMT markers in ESCC cells treated with CLDN3 gene silencing or CLDN3 gene silencing plus capsaicin; D) Comparison of protein expression of Cldn3 and EMT markers in ESCC 
cells treated with CLDN3 gene silencing or CLDN3 gene silencing plus capsaicin. All data were presented as mean $\pm S D$ of three independent experiments. Statistical analysis was performed using an unpaired Student's $t$ test. *, $\mathrm{P}<0.05 ; * \star, P<0.01$. Scale bar equals to $50 \mu \mathrm{m}$.
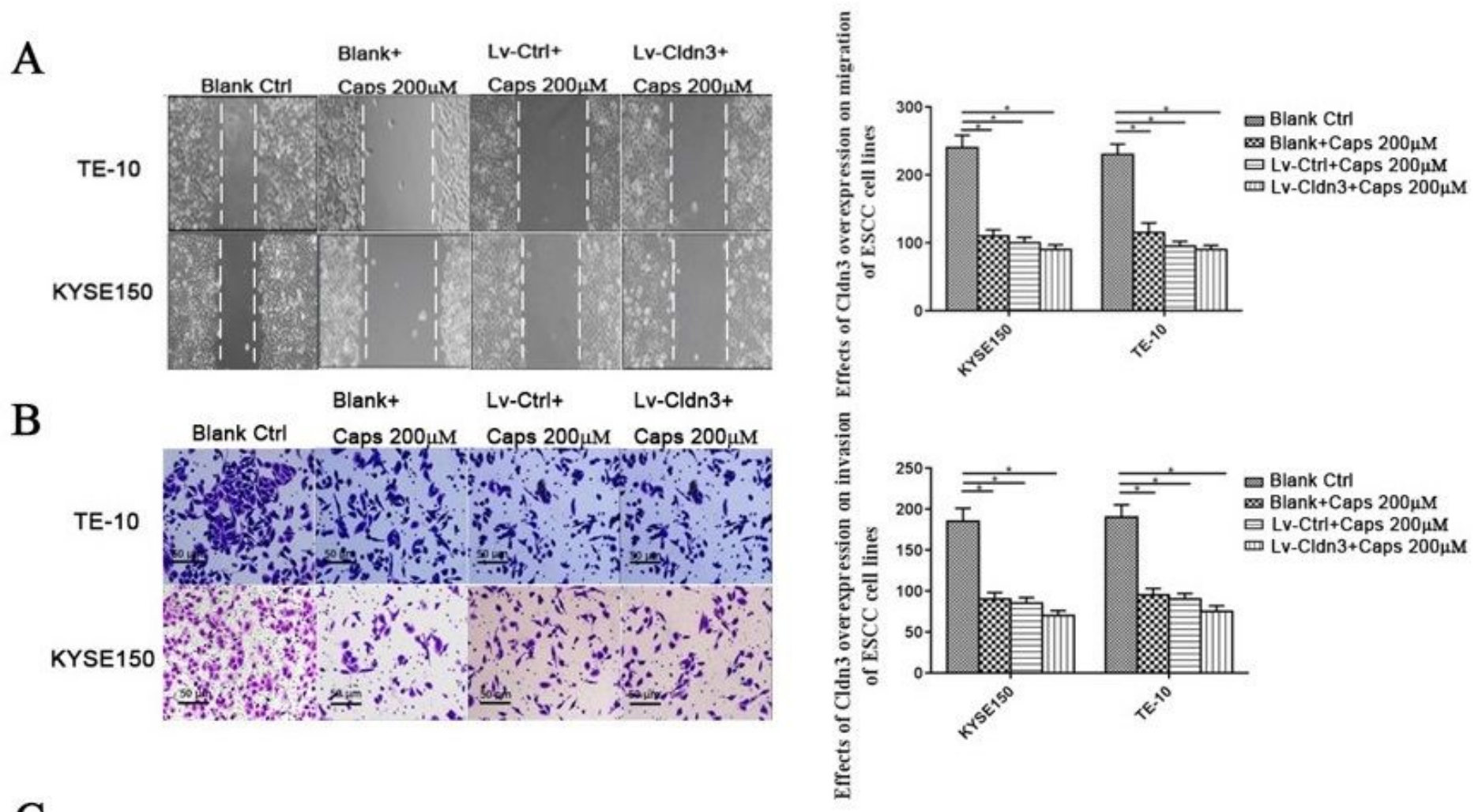

$\mathrm{C}$
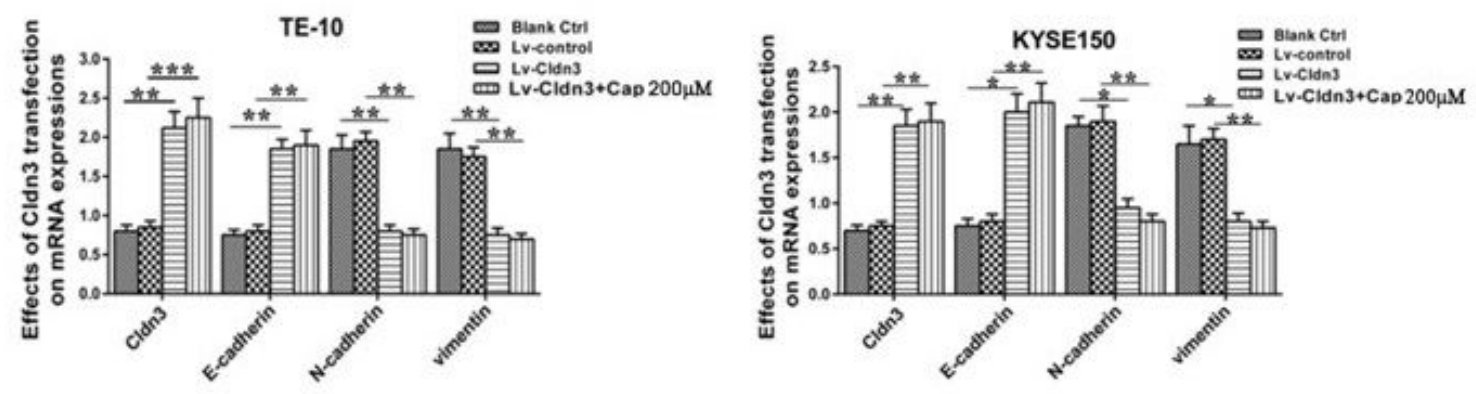

D
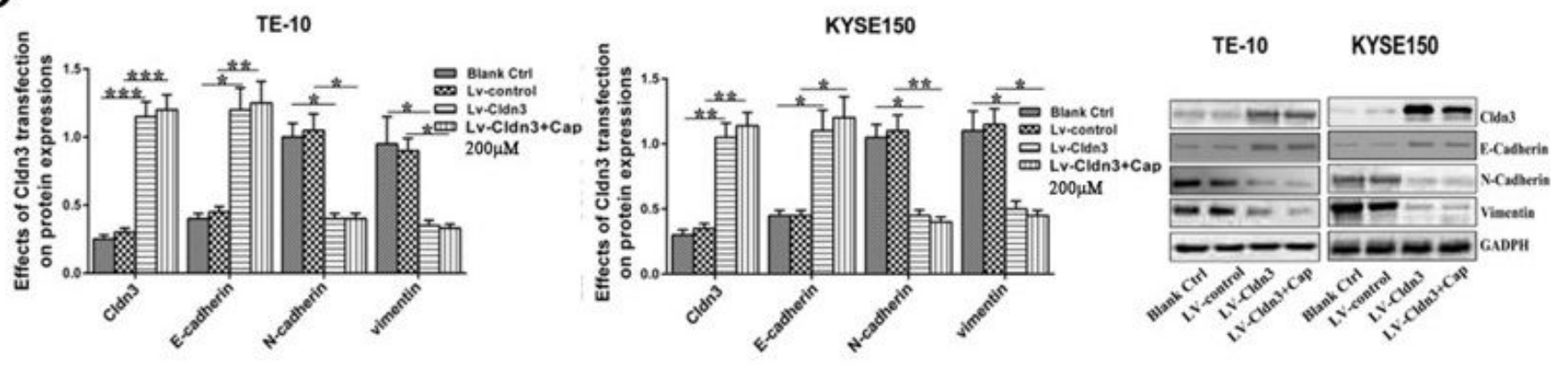

\section{Figure 5}

Effects of Cldn3 overexpression on migration and invasion of ESCC cells and the expression of EMT markers A) Comparison of migration distance of ESCC cells treated with capsaicin or Cldn3 
overexpression plus capsaicin by wound healing assay; B) Comparison of invaded ESCC cells treated with capsaicin or Cldn3 overexpression plus capsaicin by Transwell assay; C) Quantification of mRNA expression of Cldn3 and EMT markers in ESCC cells treated with Cldn3 overexpression or Cldn3 overexpression plus capsaicin; D) Comparison of protein expression of Cldn3 and EMT markers in ESCC cells treated with Cldn3 overexpression or Cldn3 overexpression plus capsaicin. All data were presented as mean $\pm S D$ of three independent experiments. Statistical analysis was performed using an unpaired Student's $t$ test. *, $P<0.05 ; * \star, P<0.01 ; \star \star \star, P<0.001$. Scale bar equals to $50 \mu \mathrm{m}$.

A

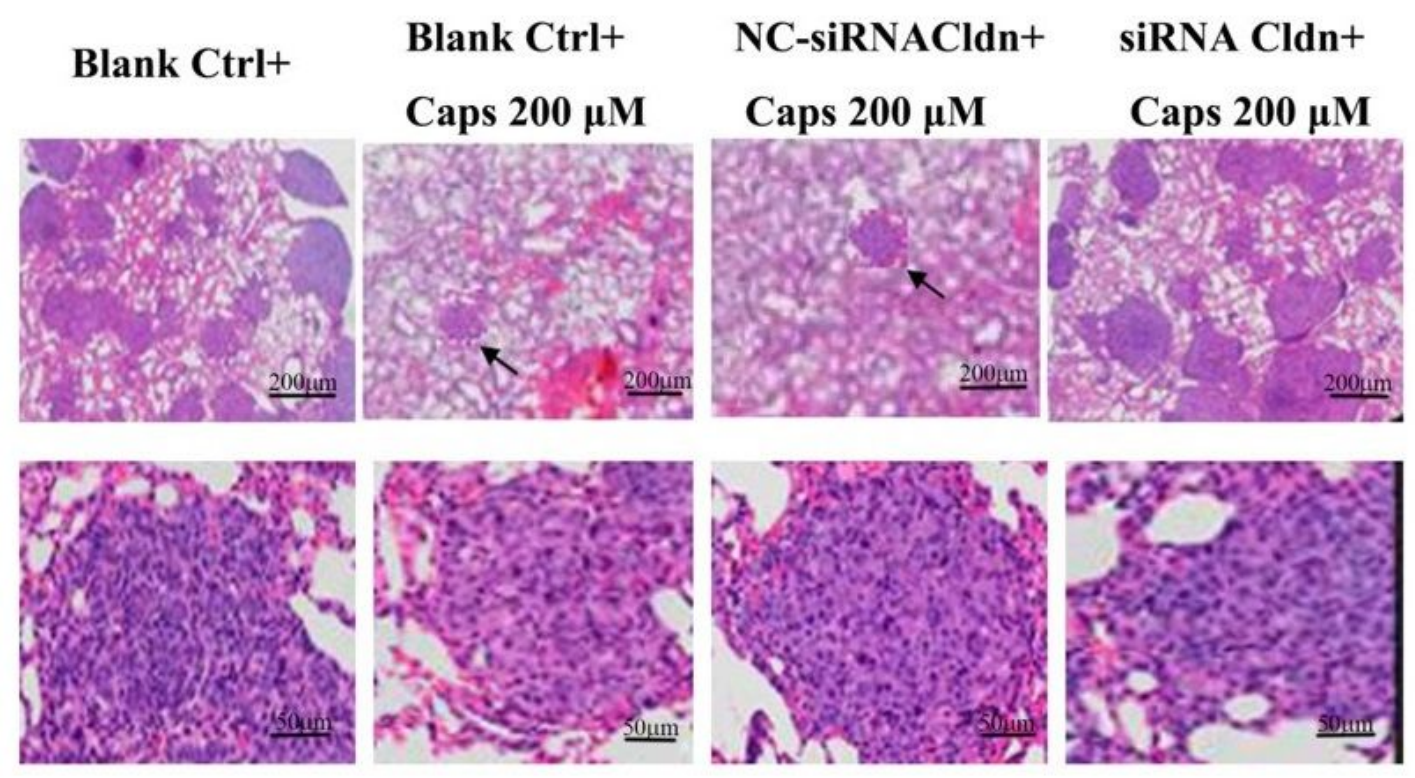

$\mathrm{B}$

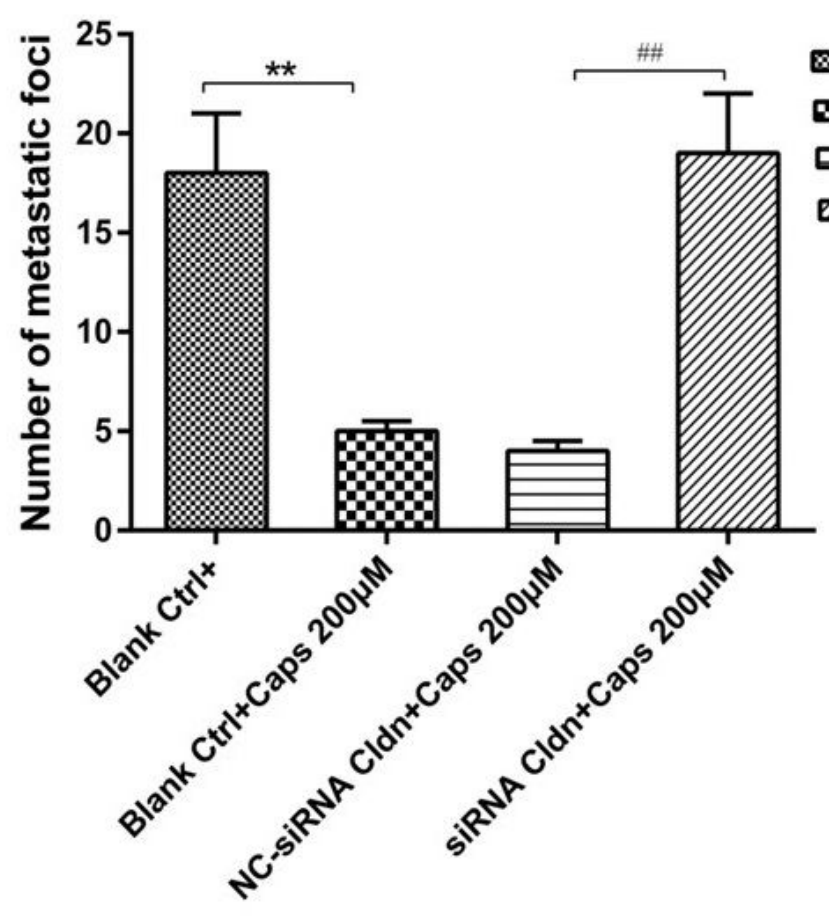

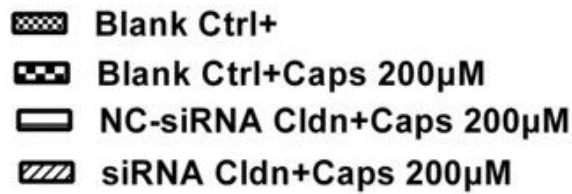


Figure 6

Effects of Capsaicin on lung metastasis of ESCC in nude mice A) Lung metastatic nodules of ESCC by hematoxylin-eosin staining; B) Quantification of lung metastatic nodules of ESCC. All data were presented as mean \pm SD of three independent experiments. Statistical analysis was performed using an

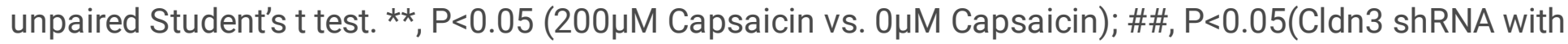
$200 \mu$ M Capsaicin vs. NC shRNA with $200 \mu \mathrm{M}$ Capsaicin). Scale bars equal to $50 \mu \mathrm{m}$ and $200 \mu \mathrm{m}$ 